\title{
ARTICLE OPEN \\ An efficient and compact switch for quantum circuits
}

Yulin Wu' ${ }^{1}$, Li-Ping Yang ${ }^{2}$, Ming Gong $\mathbb{D}^{1}$, Yarui Zheng ${ }^{1}$, Hui Deng ${ }^{1}$, Zhiguang Yan ${ }^{1}$, Yanjun Zhao ${ }^{3}$, Keqiang Huang ${ }^{4}$, Anthony D. Castellano ${ }^{1}$, William J. Munro $\mathbb{1}^{5,6}$, Kae Nemoto ${ }^{6}$, Dong-Ning Zheng ${ }^{4,7}$, C. P. Sun ${ }^{2,8}$, Yu-xi Liu ${ }^{3,9}$, Xiaobo Zhu ${ }^{1,8}$ and Li Lu $^{4}$

The engineering of quantum devices has reached the stage where we now have small-scale quantum processors containing multiple interacting qubits within them. Simple quantum circuits have been demonstrated and scaling up to larger numbers is underway. However, as the number of qubits in these processors increases, it becomes challenging to implement switchable or tunable coherent coupling among them. The typical approach has been to detune each qubit from others or the quantum bus it connected to, but as the number of qubits increases this becomes problematic to achieve in practice due to frequency crowding issues. Here, we demonstrate that by applying a fast longitudinal control field to the target qubit, we can turn off its couplings to other qubits or buses. This has important implications in superconducting circuits as it means we can keep the qubits at their optimal points, where the coherence properties are greatest, during coupling/decoupling process. Our approach suggests another way to control coupling among qubits and data buses that can be naturally scaled up to large quantum processors.

npj Quantum Information (2018)4:50 ; doi:10.1038/s41534-018-0099-6

\section{INTRODUCTION}

Superconducting quantum circuits $^{1-4}$ are promising candidates to realize quantum processors and simulators. They have been used to demonstrate various quantum algorithms and implement thousands of quantum operations within their coherence time, ${ }^{5}$ in which controllable couplings are inevitable. The typical way to couple/decouple two superconducting quantum elements with always on coupling is to tune their frequencies in or out of resonance, ${ }^{6-12}$ or with sideband transitions by parametric driven. ${ }^{13,14}$ These methods are widely adopted even for the most recent universal gate implementations. ${ }^{5,15-26}$ However, they suffer from several issues, namely, it is technically difficult to avoid the frequency crowding problem in large-scale circuits; the qubits cannot always work at the coherent optimal point; and the fast tuning of the qubit frequency results in nonadiabatic information leakage. To overcome the above problems, significant effort both theoretically and experimentally ${ }^{27-47}$ has been devoted to develop coupling methods for parametrically tuning the coupling strength between two quantum components. These proposals or demonstrations either only achieved low fidelity or needs additional circuity to modify the qubit architecture, increasing the complexity of the circuity and bring in additional cross-talk problems, which in the process of scaling up, might be a major hinderance. Therefore, the implementation of a switch for coherent coupling between quantum elements is still a big challenge in scalable quantum circuits.

In this article, we propose and demonstrate a simple architecture-independent method to switch on/off the coupling between two quantum elements via a longitudinal control field. ${ }^{48-}$
${ }^{51}$ We demonstrate this on two different superconducting systems: transmon qubits ${ }^{52}$ of the Xmon variety, ${ }^{53}$ and flux qubit-resonator systems. ${ }^{54}$ In the first instance (Fig. $1 \mathrm{a}-\mathrm{c}$ ) two qubits $Q_{1}$ and $Q_{2}$ are coupled through the same coplanar wave guide resonator with coupling strength $g_{1} \approx g_{2} \approx 20 \mathrm{MHz}$, respectively. The qubit frequencies $\omega_{1}$ and $\omega_{2}$ are lower than the resonator frequency $\omega_{c}=5.456 \mathrm{GHz}$ with detuning $\Delta_{1,2}=\omega_{c}-\omega_{1,2}$. In this dispersive limit, the Hamiltonian of the qubit-qubit system can be written as

$$
H_{\mathrm{X}}=\frac{\hbar}{2} \omega_{1} \sigma_{z, 1}+\frac{\hbar}{2} \omega_{2} \sigma_{z, 2}+\hbar g\left(\sigma_{+, 1} \sigma_{-, 2}+\sigma_{-, 1} \sigma_{+, 2}\right),
$$

where $g=g_{1} g_{2}\left(\Delta_{1}+\Delta_{2}\right) / 2 \Delta_{1} \Delta_{2}$ is the effective coupling strength, and $\Delta_{1,2}=\omega_{c}-\omega_{1,2}$ is the detuning of the qubit frequencies from the resonator. Here $\sigma_{x}, \sigma_{y}$ and $\sigma_{z}$ are the Pauli operators with $\sigma_{ \pm}=$ $\left(\sigma_{x} \pm i \sigma_{y}\right) / 2$.

The second system (Fig. 1d-f) can be described by the flux qubit-resonator Hamiltonian as ${ }^{55}$

$H_{\mathrm{F}}=\frac{\hbar}{2}\left(\Delta \sigma_{z, 1}+\varepsilon \sigma_{x, 1}\right)+\hbar \omega_{r} a^{\dagger} a+\hbar g\left(a^{\dagger}+a\right) \sigma_{x, 1}$,

where $a^{\dagger}(a)$ is the creation (annihilation) operator of the resonator field with the resonance frequency $\omega_{r} / 2 \pi$, and $\hbar \Delta$ is the energy gap of the flux qubit with $\hbar \varepsilon$ being the energy difference between the two persistent-current states. The qubit frequency is given by $\omega_{1}=\sqrt{\Delta^{2}+\varepsilon^{2}}$.

\section{RESULTS}

Let us focus our attention on our switch for both quantum circuits beginning with the qubit-qubit system.

\footnotetext{
${ }^{1}$ Hefei National Laboratory for Physical Sciences at the Microscale and School of Life Sciences, University of Science and Technology of China, Hefei, Anhui 230027, China; ${ }^{2}$ Beijing Computational Science Research Center, Beijing 100193, China; ${ }^{3}$ Institute of Microelectronics, Tsinghua University, Beijing 100084, China; ${ }^{4}$ Institute of Physics and Beijing National Laboratory for Condensed Matter Physics, Chinese Academy of Sciences, Beijing 100190, China; ${ }^{5}$ NTT Basic Research Laboratories, NTT Corporation, 3-1 Morinosato-Wakamiya, Atsugi, Kanagawa 243-0198, Japan; ${ }^{6}$ National Institute of Informatics, 2-1-2 Hitotsubashi, Chiyoda-ku, Tokyo 101-8430, Japan; ${ }^{7}$ CAS Center for Excellence in Topological Quantum Computation and School of Physical Sciences, University of Chinese Academy of Sciences, Beijing 100190, China; ${ }^{8}$ Synergetic Innovation Center of Quantum Information and Quantum Physics, University of Science and Technology of China Hefei, Anhui 230026, China and ${ }^{9}$ Tsinghua National Laboratory for Information Science and Technology (TNList), Beijing 100084, China

Correspondence: Xiaobo Zhu (xbzhu16@ustc.edu.cn)
}

Received: 15 March 2018 Revised: 14 July 2018 Accepted: 10 September 2018

Published online: 11 October 2018 

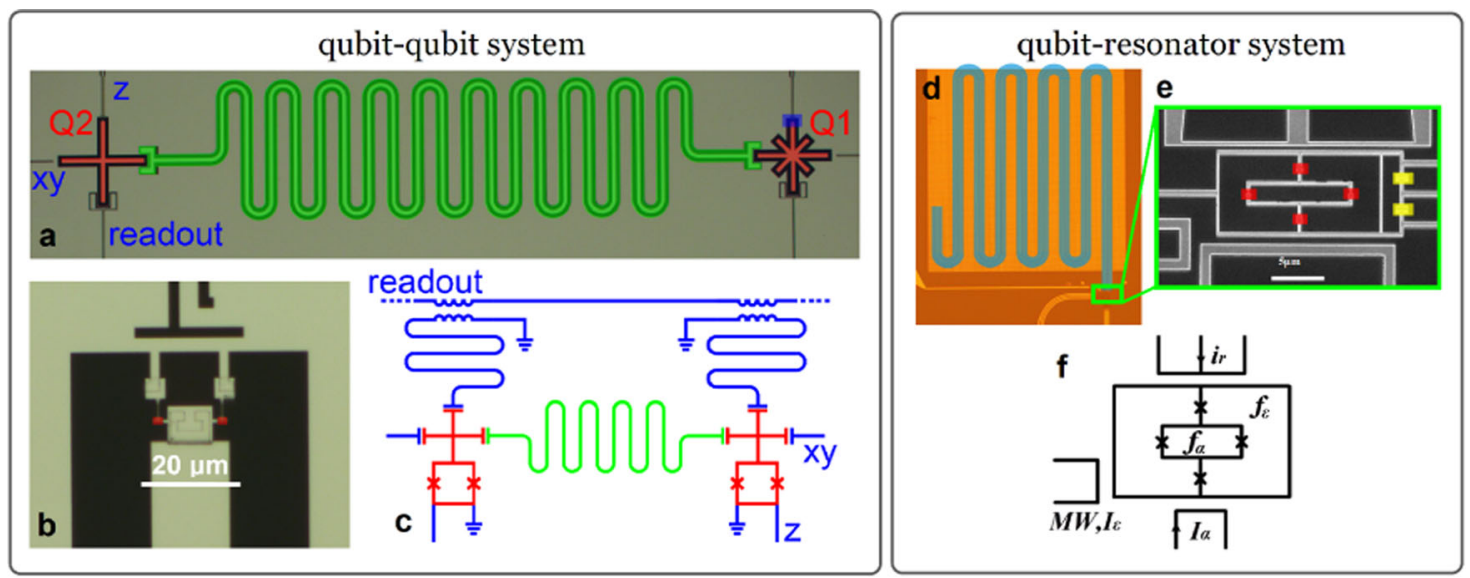

Fig. 1 The sample. Optical micrograph and schematic circuit of the qubit-qubit sample and the qubit-resonator sample. In a the two qubits $Q_{1}$ and $Q_{2}$ (shaded in red) are coupled by a coplanar wave guide resonator (shaded in green). Each qubit consists of a cross-shaped capacitor and a nonlinear inductor from a dc SQUID (shaded in blue). The dc SQUID is formed from shadow-evaporated AI Josephson junctions. A $z$ bias line inductively coupled to the dc SQUID controls the qubit frequency $\omega_{1(2)}$. The cross-shaped capacitors also serve as ports for capacitively coupling the qubit to the readout resonator, the microwave excitation line (xy), and other qubits. In $\mathbf{b}$, the small area shows the dc SQUID with the the Al Josephson junctions shaded in red, while $\mathbf{c}$ is a schematic circuit of the sample with crosses denoting the Josephson junctions. In d for the flux qubit-resonator sample, the coplanar wave guide resonator is marked out by a blue ribbon, while e shows a SEM image of the small structures of the flux qubit circuit. Josephson junctions of the qubit and the readout dc-SQUID are marked out by red and yellow boxes, respectively. A schematic illustration of the gap-tunable flux qubit with control and coupling lines is shown in $\mathbf{f}$. The smaller junction of the three-junction flux qubit is replaced by a dc-SQUID, called the $a$-loop, in which the flux $\mathrm{f}_{a}$ threading the $a$-loop is tuned by the current $I_{a}$ through the $a$ bias line. The $a$ bias line is also used for applying longitudinal control pulses ( $z$ pulses) to perform the switch. The qubit is coupled to the resonator through mutual inductance. Qubit flux bias $\mathrm{f}_{\varepsilon}$ and microwave pulses are generated by the current $I_{\varepsilon}$ through another microwave line on the left. To achieve high flux bias stability, the flux qubit adopts a gradiometeric geometry. $I_{\mathrm{r}}$ Here $i_{r}$ denotes the zero-point current of the resonator. The readout dc-SQUID is not shown here

\section{Qubit-qubit system configuration}

We begin by fixing the the qubit frequency of $Q_{2}$ at $\omega_{1}=$ $4.9174 \mathrm{GHz}$, denoted by the horizontal solid line in Fig. 2a. We then tune the qubit frequency of $Q_{1}$ by its $z$ bias line and probe its microwave excitation response. Figure $2 \mathrm{a}$ shows the spectroscopic measurement with an anticrossing due to the qubit-qubit coupling clearly observed at $4.9174 \mathrm{GHz}$. Fitting the spectrum lines (dashed blue curves in Fig. 2a) establishes the coupling strength as $g / 2 \pi=0.61 \mathrm{MHz}$.

To begin our investigation of switching on/off the qubit-qubit coupling, we apply a control field with frequency $\omega_{z} / 2 \pi=20 \mathrm{MHz}$ to $Q_{1}$ by the $z$ bias line. This results in a longitudinal interaction Hamiltonian $H_{\mathrm{L}}=\hbar \lambda_{z} \cos \left(\omega_{z} t\right) \sigma_{z, 1}$, where $\lambda_{z}$ is determined by the amplitude of the applied rf driving current $I_{a}$ in the $z$ bias line. By performing an unitary transform $U=\exp \left[-i 2 \lambda_{z} \sin \left(\omega_{z} t\right) \sigma_{z} / \omega_{z}\right]$, the total Hamiltonian $H=H_{\mathrm{JC}}+H_{\mathrm{L}}$ of the system reduces to the effective Hamiltonian of the form ${ }^{48,49}$

$H_{\text {eff }}=\frac{\hbar}{2} \omega_{1} \sigma_{z, 1}+\frac{\hbar}{2} \omega_{2} \sigma_{z, 2}+\hbar g_{\text {eff }}\left(\sigma_{+, 1} \sigma_{-, 2}+\sigma_{-, 1} \sigma_{+, 2}\right)$,

where we have neglected the small fast-oscillating terms assuming $\omega_{z} \gg g$. In this case $g_{\text {eff }}=g J_{0}\left(2 \lambda_{z} / \omega_{z}\right)$ is the effective coupling strength under longitudinal control, where $J_{0}(x)$ is the zeroth-order Bessel function of the first kind. Our effective Hamiltonian clearly shows that $g_{\text {eff }}$ vanishes when $2 \lambda_{z} / \omega_{z}$ is a zero point of the Bessel function $J_{0}\left(2 \lambda_{z} / \omega_{z}\right)$, that is when $\lambda_{z} \approx 1.2 \omega_{z}$. At this point the qubit-qubit coupling is switched off. Moreover, the coupling strength $g_{\text {eff }}$ can be continuously tuned between two values with opposite signs by changing the ratio $2 \lambda_{z} / \omega_{z}$. We can define the switch on/off ratio $R$ as the ratio between vacuum Rabi frequencies with and without the control field. Henceforth, we will call $\lambda_{\text {zoff }}$ the switch-off point and a longitudinal control pulse with such an amplitude a switch-off pulse. Here, we have neglected the fast-oscillating terms to give a simplified description to illustrate the idea of this scheme, in methods we have done numerical simulations with the full Hamiltonian (see Methods). In fact, experiment ${ }^{56}$ and theory ${ }^{49}$ have shown that transparency to the transverse classical field can be induced with a longitudinal control pulse. Here, we replace the transverse classical field with a quantum element (qubit or resonator), which becomes decoupled from all transverse interactions. This is the governing principle of our controlled coupling scheme.

For the qubit-qubit case, in view of the qubits small anharmonicities around $250 \mathrm{MHz}$, we included one extra level in our numerical simulations. Results are consistent with that of the analytical two-level case described by Eq. (3). The driving amplitude used for the qubit-qubit system in this work is well below the qubit anharmonicity and leakage to higher levels is well below $10^{-4}$. Note that if the amplitude approaches the anharmonicity, leakage will become nonnegligible.

Next, to test our longitudinal, control field-based switch we perform spectroscopic measurements on the qubit-resonator system under different amplitudes $\lambda_{z}$ of the longitudinal control fields. These are shown in Fig. $2 b$ where it can be seen that the amplitude of the anticrossing gap $\Delta_{g}=2 g_{\text {eff }}$ decreases to zero as $\lambda_{z} \rightarrow 1.2 \omega_{z}$ and then opens up again as $\lambda_{z}$ further increases. At the switch-off point $\lambda_{\text {zoff }} \approx 24 \mathrm{MHz}$, the amplitude of the anticrossing $\Delta_{g}$ becomes undetectable. This is definite evidence that the coupling can be tuned and switched off by the longitudinal control field.

\section{Qubit-resonator system configuration}

Here, we tune the qubit energy gap $\Delta / 2 \pi$ to be equal to the resonator frequency $\omega_{r} / 2 \pi=2.417 \mathrm{GHz}$ by applying a long dc bias in the $a$ bias line (see Fig. 1c). Spectroscopic measurements are then performed. Figure $2 \mathrm{c}$ shows the measurement results with an anticrossing due to the qubit-resonator coupling clearly observed at $2.417 \mathrm{GHz}$. Fitting the spectrum lines (dashed blue curves) allows us to determine the coupling strength as $g / 2 \pi=9.14 \mathrm{MHz}$.

Given we now know $g$ we can now test our switching protocol. Applying a longitudinal control field (similar to the qubit-qubit case) with frequency $\omega_{z}$ and amplitude $\lambda_{z}$, we observed in Fig. $2 d$ that the amplitude of the anticrossing gap $\Delta_{g}=2 g_{\text {eff }}$ decreases to 

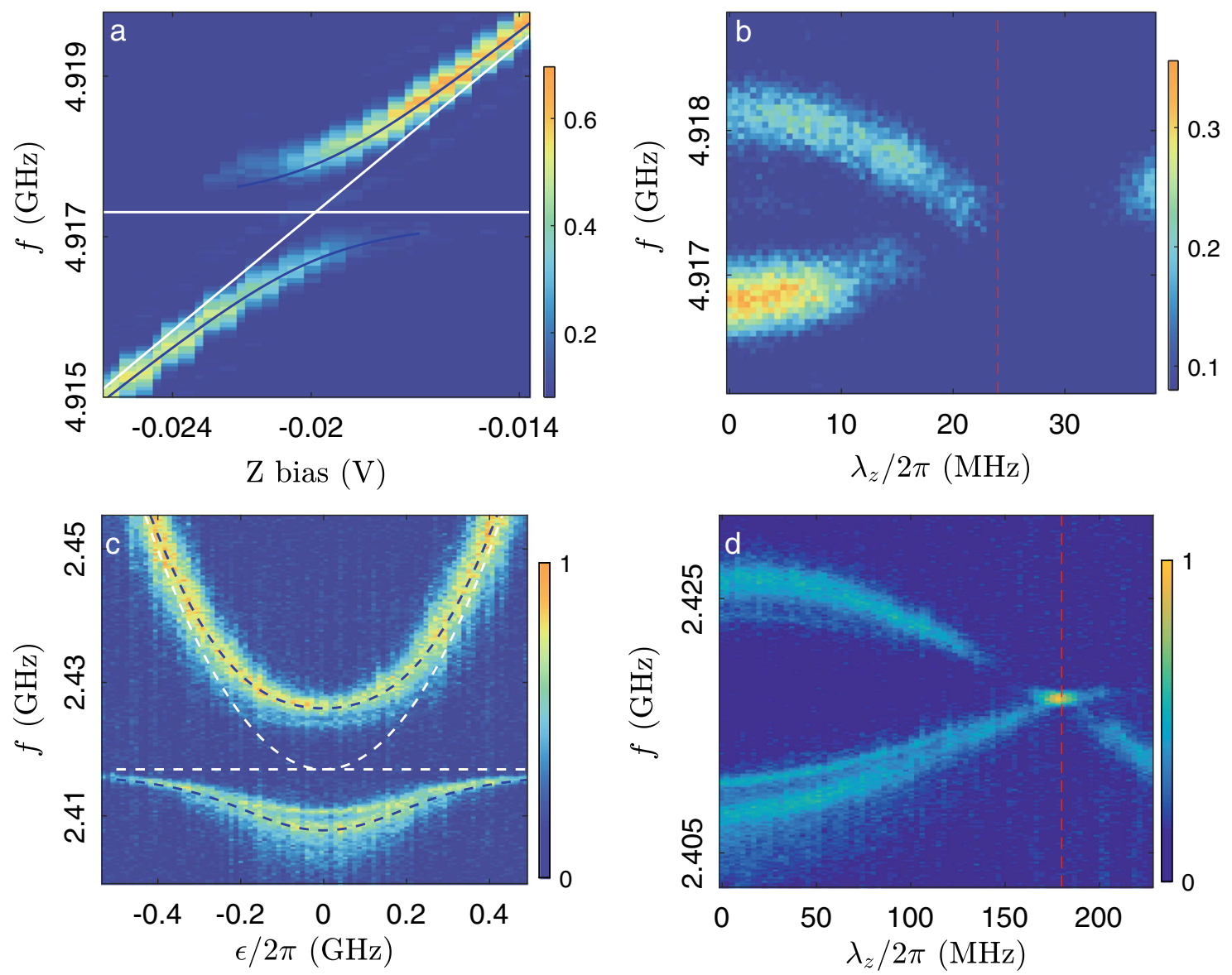

Fig. 2 Spectrum of the qubit-qubit and the qubit-resonator systems. In a the spectrum of qubit $Q_{1}$ in the qubit-qubit system is displayed. The qubit is driven by a microwave pulse with varied frequency $f$, which when $f$ matches the qubit frequency $\omega_{1}$ excites the qubit to its exited state. This increases the excited state occupation probability $P$. The qubit frequency can then be tuned by a $z$ bias line. With the frequency $\omega_{2} /$ $2 \pi$ of the second qubit $Q_{2}$ fixed at $4.9174 \mathrm{GHz}$ (indicated by the horizontal white dashed line) an anticrossing gap $\Delta_{g}=2 \mathrm{~g} / 2 \pi=1.22 \mathrm{MHz}$ of the spectrum lines due to the coupling is clearly visible. Also show as a diagonal white line are the qubit frequencies of $Q_{1}$ with zero coupling strength. Further the blue solid curves are fit of the spectrum lines. Next under the longitudinal drive with frequency $\omega_{z} / 2 \pi=20 \mathrm{MHz}$ the anticrossing gap $\Delta_{g}\left(\lambda_{z}\right)$ variation is depicted in $\mathbf{b}$ with the amplitude $\lambda_{z}$ of the control pulse. $\Delta_{g}$ decreases when increasing $\lambda_{z}$ from zero, and reaches an invisible minimum value at the switch-off point $\lambda_{z}=\lambda_{z \text { off }} \approx 1.2 \omega_{z}$ indicated by the vertical dashed line. In $\mathbf{c}$ the spectrum of the flux qubit $Q_{1}$ in the qubit-resonator system is displayed as a function of $\varepsilon$. The qubit is driven by a microwave pulse with variable frequency $f$, and when $f$ matches the qubit frequency $\omega_{a b}$ the qubit is excited to its exited state. The frequency $\omega_{r} / 2 \pi$ of the resonator is $2.417 \mathrm{GHz}$, indicated by the horizontal white dashed line, while the other white dashed line shows the qubit frequencies at zero coupling strength. The blue dashed curves are fit of the spectrum lines. Clearly shown is an anticrossing due to the coupling with gap $\Delta_{g}=2 g / 2 \pi=18.28 \mathrm{MHz}$. In d the anticrossing gap $\Delta_{g}\left(\lambda_{z}\right)$ is shown in varying amplitude $\lambda_{z}$ of the control pulse with frequency $\omega_{z} / 2 \pi=150 \mathrm{MHz}$ at the qubit optimal point. $\Delta_{g}$ decreases when increasing $\lambda_{z}$ from zero, and reaches a minimum value at the switch-off point $\lambda_{z}=\lambda_{\text {zoff }} \approx 1.2 \omega_{z}$ indicated by the vertical dashed line, and then reopened again to about $0.4 \mathrm{~g}$. The color bars in $(\mathbf{a}, \mathbf{b})$ is the occupation probability of the qubit excited state and in (c, $\mathbf{d})$ is the readout signal normalized to the range of $[0,1]$

zero as $\lambda_{z} \rightarrow 1.2 \omega_{z}$. The bright spot seen at $\Delta / 2 \pi=2.417 \mathrm{GHz}$ and near $\lambda_{z} / 2 \pi \sim 180 \mathrm{MHz}$ is the resonator's resonance signature. When we drive the flux qubit, the resonator can be excited due to coupling with the qubit microwave driving control line. This excitation can be detected because there is coupling between the resonator and the qubit readout SQUID, which results in the bright spot at the resonator frequency in Fig. $2 \mathrm{~d}$.

\section{Performance}

Our exploration of the switch using spectroscopic measurements has qualitatively shown its operation in both the qubit-qubit and qubit-resonator configurations, yet it is difficult to quantify exactly how well it is operating from those measurements alone. However, time-domain vacuum Rabi oscillation measurements with the switch turned on and off separately will allow us to measure the time scale of the relaxation decay. Figure $3 a$ for the qubit-qubit system and (c) for the qubit-resonator system shows the vacuum Rabi oscillations when the amplitude $\lambda_{z}$ of the longitudinal control field is increased from zero. The data clearly shows that the oscillation frequency $\omega_{c}=2 g_{\text {eff }}$ decreases with increasing $\lambda_{z}$. When $\lambda_{z}$ reaches the switch-off point $\lambda_{z \text { off, the }}$ oscillation frequency reaches a minimum. Further, Fig. $3 b$ for the qubit-qubit system and $(d)$ for the qubit-resonator system shows the comparison of the dynamics without a control pulse (blue) and with switch-off pulse (orange). At the switch-off point the dynamic behavior is an exponential decay-the oscillation frequency is too small to be observed on the experimental data, indicating a very small effective coupling $g_{\text {eff. The rate of }}$ exponential decay due to energy relation in the switched-off state is very close to the decay rate without the switching pulse (shown explicitly in Fig. 3b for the qubit-qubit system). This indicates that the switching pulse does not influence the qubits relaxation time. More quantitatively, the characteristic times for the decays in Fig. 3b are 

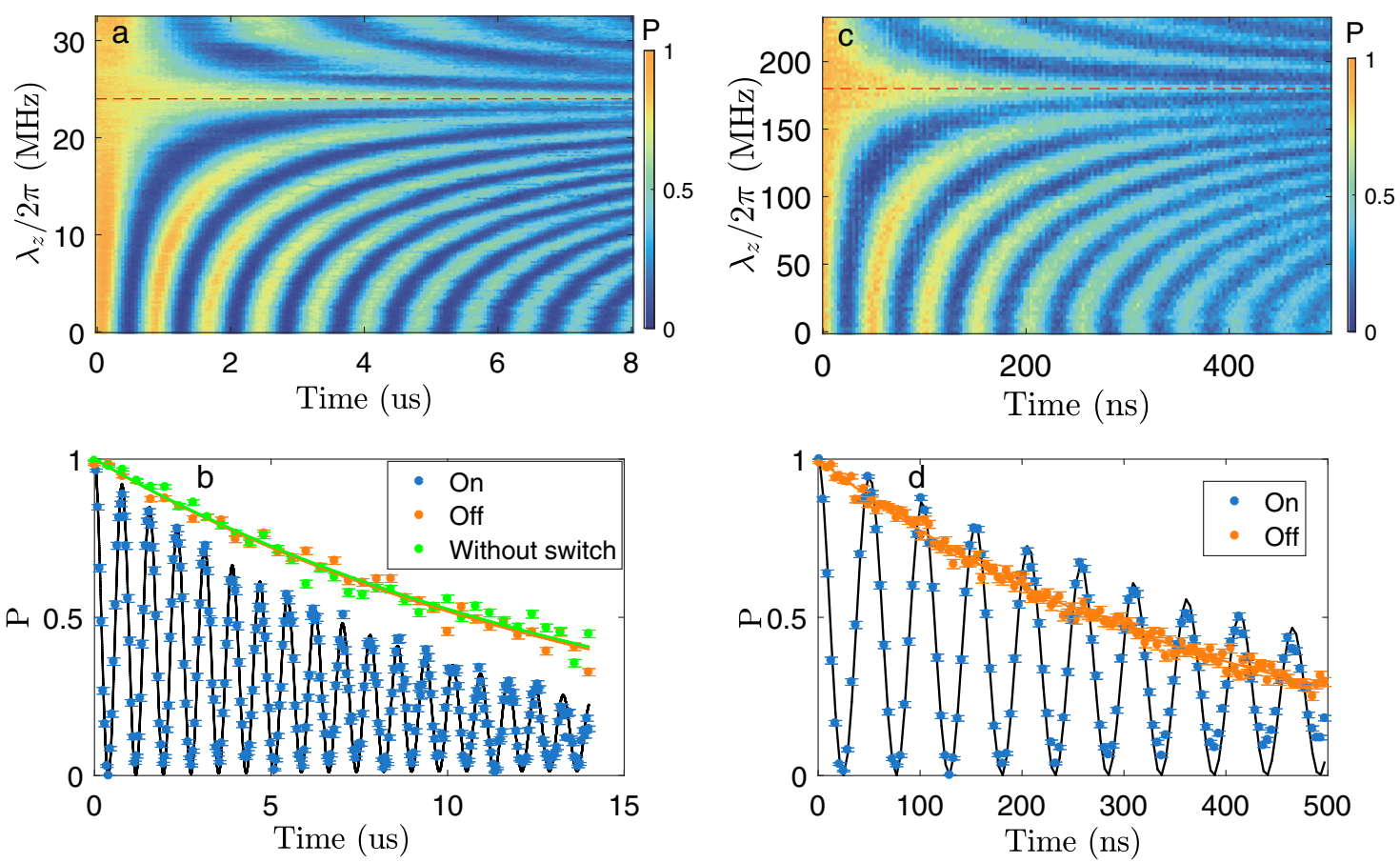

Fig. 3 Switching on/off the coherent oscillation between the qubit-qubit and the qubit-resonator systems. Vacuum Rabi oscillations between the qubit and the resonator under different amplitudes $\lambda_{z}$ of longitudinal control pulse for the qubit-qubit a and qubit-resonator $\mathbf{c}$ configurations. When $\lambda_{z}$ is increased, the oscillation frequency decreases and reaches a minimum at the switch-off point $\lambda_{z}=\lambda_{z o f f}$ indicated by a red dashed line. The color indicates the occupation probability $P$ of the qubit excited state. This is consistent with the numerical simulation in Fig. 5 c. In $\mathbf{b}$ for the qubit-qubit and $\mathbf{d}$ for qubit-resonator situations we display a comparison between the vacuum Rabi oscillation without (blue) and with (orange) longitudinal control for $\lambda_{z}=\lambda_{\text {zoff. }}$ When the longitudinal control with the amplitude $\lambda_{\text {zoff }}$ is applied to the qubit, the vacuum Rabi oscillation between the qubit and the resonator vanishes, indicating that the qubit-resonator coupling is switched off. Dots are experimental data, solid curves are both exponential decay fit and exponential decay oscillation fit. As a comparison in the qubit-qubit situation, the qubit decay without switching pulse is also plotted (green dots). Here we observe that the qubit decays at the same rate in both cases. We have also compared $T_{2}^{*}$ with and without longitudinal driving and found no noticeable degradation of $T_{2}^{*}$
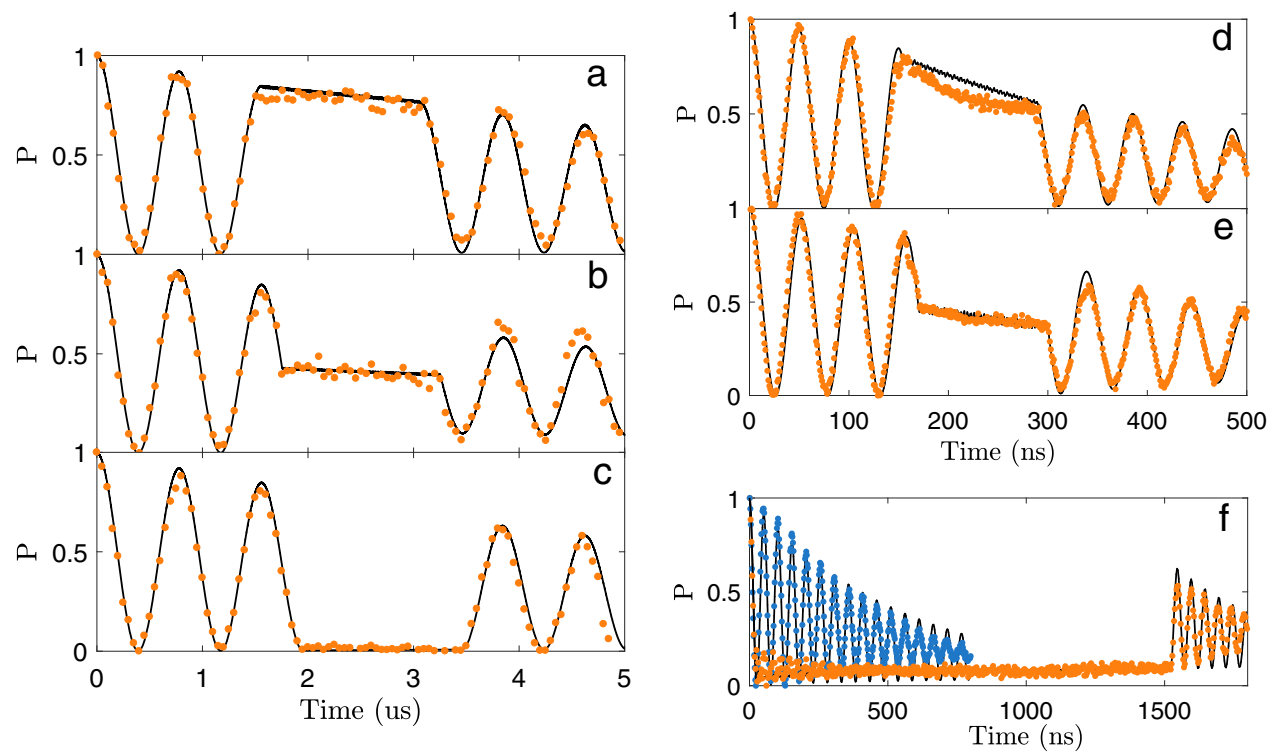

Fig. 4 Dynamics of switching on/off the coupling. The qubit $Q_{1}$ is initially prepared in its excited state and allowed to interact with either the second qubit (in the case of the qubit-qubit configuration) or the resonator (in the flux qubit configuration). At a certain time, a switch-off pulse is applied, freezing the coherent oscillation between the two systems. At a later time, the switching pulse is removed and coherent oscillation resumes. In $\mathbf{a}$, $\mathbf{d}$ the switch-off pulse is applied when the first qubit is in its excited state, while in $\mathbf{b}$, $\mathbf{e}$ the coupling is switched off when $Q_{1}$ and either $Q_{2}$ or the resonator are in an entangled state. Finally, for $\mathbf{c}, \mathbf{f}$ the coupling is switched off when $Q_{1}$ is in the ground state. Solid lines are simulations with measured energy relaxation times, $15.6 \mu \mathrm{s}$ for $Q_{1}$ and $7.0 \mu \mathrm{s}$ for $Q_{2}$ in the qubit-qubit system, $0.45 \mu \mathrm{s}$ for the qubit and $4.6 \mu \mathrm{s}$ for the resonator in the flux qubit system. a-c is the data for the qubit-qubit system while d-f is the data for the qubitresonator system 
1. Single-qubit energy relaxation rate: $T=15.58 \pm 0.28 \mu \mathrm{s}$

2. No switching pulse with maximal coupling: $T=9.57 \pm$ $0.35 \mu \mathrm{s}$

3. Switching pulse tuned to turn off the coupling: $T=15.25 \pm$ $0.24 \mu \mathrm{s}$

We observed that the difference between the "No switching pulse with maximal coupling" and "Switching pulse tuned to turn off the coupling" situations is 9.57 compared with 15.25 for the " 2 " and " 3 " situations, this is because the relaxation time of $Q_{2}$ is $7.0 \mu \mathrm{s}$, shorter than that of $Q_{1}(15.58 \mu \mathrm{s})$. Further, it is quite interesting that the decay behavior between the " 1 " and " 3 " situations are almost the same, even if we attribute all the slight difference to a weak, residual coupling, it should be less than $2.7 \mathrm{kHz}$ (see Methods). The lower limit of the on/off ratio is $g / g_{r}=$ $0.61 \mathrm{MHz} / 2.7 \mathrm{kHz} \sim 230$, where $g$ is the coupling strength between the two qubits. Further, the on/off ratio could be significantly enhanced by improving the qubit coherent properties and the precision of the control pulse, since the effective coupling $g_{\text {eff }}=$ $g J_{0}\left(2 \lambda_{z} / \omega_{z}\right)$ can be tuned from positive to negative crossing the zero point. Also, as can be seen in the Methods section (Fig. 6) the numerical simulation shows an on-off ratio higher than $10^{5}$, which hightlights the potential of the longitudinal control field as a highefficiency way to turn off the coupling.

While we have shown that our longitudinal pulse enables an effective switching operation, it is important to establish its effect on the quantum coherence of the systems. To this end we will now demonstrate the dynamical switching on and off of the coupling. In this case a target qubit is initially prepared in its excited state and brought into coherent resonance with its corresponding quantum element, after a delay, a switch-off pulse is applied over a period of time. In Fig. $4 a, d$, the coupling is switched off when the target qubit is in the excited state. In Fig. $4 b$, e, the coupling is switched off when the system is in an entangled state. In Fig. 4c, f, the coupling is switched off when the target qubit is on the ground state. We found that regardless of what state the system is in, when the switch-off pulse is applied, the coherent oscillation is paused (except for free evolution and decay during the switch-off time interval), and when the switch-off pulse is removed, the coherent oscillation resumes.

\section{DISCUSSION}

We have demonstrated a highly efficient switch with an if control field, the coupling strength can be in principle tuned to zero in both a qubit-qubit system and a qubit-resonator system. Dynamically switching on and off the coupling is also shown. The on-off ratio was measured to be above 230, and could be significantly improved in the future. In principle, the coulping could also be modified from $g$ to any value in the range $[-0.4 g, g]$, which allows the system dynamics to be retarded or reversed. This could be useful in spin-echo-like refocusing appliactions. This type of switch scheme can be applied to any qubit system with longitudinal control field and scales easily since no auxiliary circuit is needed. For applications to $N$ qubits, we have done a preliminary investigation for cases with nearest-neighboring coupling. For the case of 1-D chain qubits with nearestneighboring coupling, our numerical simulations show that by applying driving fields on every other qubit, couplings can be switched off when all $N$ qubits are at the same qubit frequency. Scaling up to 2-D configurations is also straightforward. We also point out that besides as a tunable coupling scheme between quantum elements, the longitudinal coupling and control between electromagnetic fields and quantum devices itself is of great interest. $^{56-63}$

\section{METHODS}

The qubit-qubit sample

This sample consists of six qubits fabricated with aluminum evaporation and lift-off techniques. The Josephson junctions are formed by using the Dolan bridge technique. The two qubits on which we perform the experiments are interconnected by a coplanar resonator, which couples them dispersively, the resonator has a resonance frequency about $5.45 \mathrm{GHz}$, well above the qubit frequencies. The $T_{1}$ of the two measured qubits are approximately 14 and $7 \mu \mathrm{s}$, while $T_{2}^{*}$ is $\sim 7 \mu$ s for both qubits.

\section{The qubit-resonator system}

The sample is a gap-tunable flux qubit inductively coupled to a $\lambda / 4$ coplanar resonator. A flux qubit is a superconducting loop interrupted by three Josephson junctions. Two of them are of the same size, and the other is smaller by a factor of $a(a<1)$. The larger two are characterized by Josephson energy $E_{J}$ and charge energy $E_{c}$. The qubit states correspond to clockwise and counter-clockwise persistent currents $\pm I_{p}$ in the qubit loop, $I_{p} \approx 400 \mathrm{nA}$ in our sample. When the magnetic flux bias in the qubit loop $\Phi_{\varepsilon}=\Phi_{0} / 2$, called the optimal point, the two persistent-current states are degenerate, quantum tunneling lifts this degeneracy, forming an energy gap $\hbar \Delta$. Once $E_{J}$ and $E_{c}$ are fixed, the energy gap of a flux qubit is determined by $a$, the ratio of critical current between the smaller and larger junctions. Since the resonator frequency is fixed, and since there exists an optimal point of coupling between the qubit and resonator where the coherence properities of the system are the best, it is desirable to have the energy gap tunable. In our sample, the smaller junction has been replaced by a dc-SQUID, called the the $a$-loop, making the energy gap tunable in a wide range. The longitudinal control pulse for switching on/off the coupling is also applied to the qubit through the a-loop. To achieve high magnetic flux bias stability, we use a gradiometric design for the qubit loop, making the qubit insensitive to global flux fluctuations. The qubit state is detected by a readout dc-SQUID inductively coupled to the qubit. The sample is fabricated on a silicon wafer using electron beam lithography patterning, aluminum evaporation, and lift-off techniques. Josephson junctions are formed by using the Dolan bridge technique. The relaxation times of the qubit and the resonator are 0.45 and $4.6 \mu \mathrm{s}$, respectively.

\section{Determination of the measured switch efficiency}

In the case of the "Single-qubit energy relaxation" of Fig. 3b (Case 1), the relaxation time was measured as $T=15.58 \pm 0.28 \mu \mathrm{s}$, while in the case of the "switching pulse tuned to turn off the coupling" (Case 3 ), the relaxation time was $T=15.25 \pm 0.24 \mu \mathrm{s}$. Using the worst case after $14 \mu \mathrm{s}$ decay, the $95 \%$ confidence upper bound of case 1 and the lower bound of case 3 , the population ratio between case 3 and case 1 is 0.89 . If we attribute the entire population drop to the residual coupling, the upper limit of oscillation frequency induced by the residual coupling is $2 g_{r}=\arccos$ $(0.89) /(14 \mu \mathrm{s} / 2 \pi)=2.7 \mathrm{kHz}$.

Model of the qubit-qubit system

Our full system can be described by the Hamiltonian

$H(t)=H_{0}+H_{l}+H_{L}$,

with

$H_{0}=\frac{\hbar}{2} \omega_{1} \sigma_{z, 1}+\frac{\hbar}{2} \omega_{2} \sigma_{z, 2}$

$H_{l}=\hbar g\left(\sigma_{+, 1} \sigma_{-, 2}+\sigma_{-, 1} \sigma_{+, 2}\right)$,

$H_{L}=\hbar \lambda_{z} \sigma_{z, 1} \cos \omega_{z} t$

Here, $H_{0}$ describes the free Hamiltonian of the two qubits with $\sigma_{z, i}=$ $\left|e_{i}\right\rangle\left\langle e_{i}|-| g_{i}\right\rangle\left\langle g_{i}\right|$ being the Pauli matrix with the excited $\left|e_{i}\right\rangle$ and ground $\left|g_{i}\right\rangle$ states of the $i$ th qubit. The interaction between the qubits is given by $H_{l}$, where $g$ is the qubit-qubit coupling strength. Finally, the longitudinal driving field applied to qubit 1 is given by the Hamiltonian $H_{L}$ where $\lambda_{z}$ is the amplitude of that field. We will also assume the coupling strength $g$ is much smaller than the frequency of the control field $\left(g \ll \omega_{z}\right)$. 

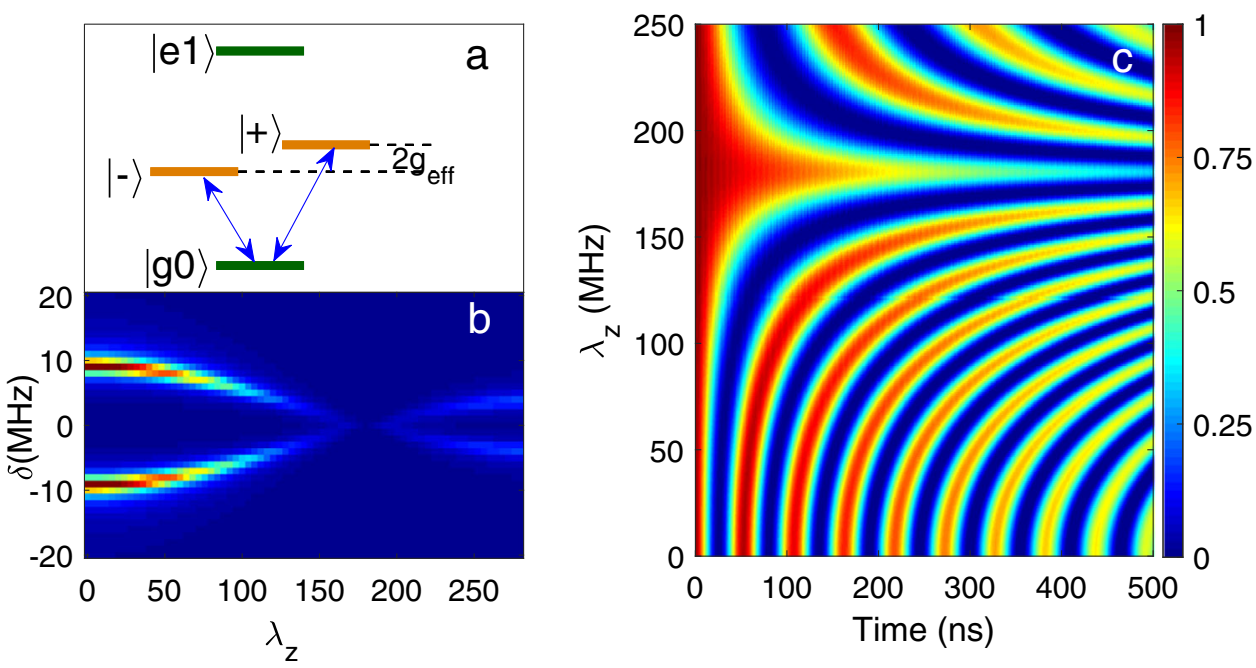

Fig. 5 a The schematic of the four lowest energy levels of the coupled system is shown. The energy gap between states $| \pm\rangle$ is $2 g_{\text {eff }}$ if the qubit is resonant with the resonator, i.e., $\omega_{a b}=\omega_{r}$. $\mathbf{b}$ The absorption spectrum of the coupled system is presented. The two absorption peaks correspond to two resonant transitions, denoted by the blue arrows in $\mathbf{a}$. The strength of the coupling between the qubit and the resonator is about $g / 2 \pi=9.14 \mathrm{MHz}$, and the frequency of the controlling field is $\omega_{z} / 2 \pi=150 \mathrm{MHz}$. The decay rates of the qubit and the resonator are about $\gamma_{q b}=0.35 \mathrm{MHz}$ (corresponding $T_{1}=450 \mathrm{~ns}$ ) and $\gamma_{r}=0.035 \mathrm{MHz}$ (corresponding $T_{1}=4.6 \mu \mathrm{s}$ ), respectively. c The occupation probability $P$ of the qubit vs. time and the strength $\lambda_{z}$ of the control field are displayed. Here, the system is initialized in the state $|e, 0\rangle$. When no control field is applied $\left(\lambda_{z}=0\right), P$ oscillates with time in period $T_{R}=2 \pi / g$ (Rabi oscillation). The control field diminishes the effective coupling strength and elongates the period of the Rabi oscillation. At the switch point, the coupling between the qubit and resonator is effectively switched off and the Rabi oscillation disappears. When $\lambda_{z}$ goes beyond this point, the coupling is switched on again and Rabi oscillation recovers

Moving to an interaction picture with respect to $H_{0}+H_{L}$, our resulting Hamiltonian can be written in the form

$$
\begin{aligned}
& H_{l}=\hbar g\left[\exp \left(i \frac{2 \lambda_{z}}{\omega_{z}} \sin \omega_{z} t\right) \sigma_{+, 1} \sigma_{-, 2}+\text { h.c. }\right], \\
& =\hbar g \sum_{n=-\infty}^{\infty}\left[J_{n}\left(\frac{2 \lambda_{z}}{\omega_{z}}\right) e^{i n \omega_{z} t} \sigma_{+, 1} \sigma_{-, 2}+\text { h.c. }\right],
\end{aligned}
$$

where we have used the Jacobi-Anger expansion and $J_{n}(x)$ is the Bessel function of the first kind. As $g J_{n \neq 0}\left(2 \lambda_{z} / \omega_{z}\right) \ll \omega_{z}$, the nonresonant terms $(n \neq 0)$ can be neglected and the interaction between the qubits is reduced to

$H_{l} \approx \hbar g_{\text {eff }} \sigma_{+, 1} \sigma_{-, 2}+$ h.c.,

with the effective coupling strength $g_{\text {eff }}=g J_{0}\left(2 \lambda_{z} / \omega_{z}\right)$. When the ratio $2 \lambda_{z} /$ $\omega_{z}$ reaches the first zero point of the Bessel function $J_{0}(x)$ near $x \approx 2.4$, the qubits are effectively decoupled. We call this point $\lambda_{z \text {,off }}$ the switch-off point. The fast oscillation terms neglected above result in a small oscillation on the qubit-qubit state when the qubit- qubit coupling is switched off. The amplitude of this oscillation decreases rapidly with increasing $\omega_{z}$.

Model for the flux qubit-resonator system

The derivation of an effective Hamiltonian of the form

$H_{l} \approx \hbar g J_{0}\left(2 \lambda_{z} / \omega_{z}\right) \sigma_{+, 1} a+$ h.c.,

follows in a similar fashion to the qubit-qubit systems derivation where one simply replaces the second qubit $\frac{\hbar}{2} \omega_{2} \sigma_{z, 2}$ with a harmonic oscillator $\hbar \omega_{r} a^{\dagger} a$ and that qubit's raising and lower operators with the bosonic creation/destruction operators.

\section{Numerical simulations}

The time evolution of our system including dissipation can be described by the Lindblad Markov master equation

$$
\frac{d \rho}{d t}=-\frac{i}{\hbar}[H, \rho(t)]+\left(\mathcal{L}_{q b}[\rho]+\mathcal{L}_{r}[\rho]\right)
$$

$$
\begin{aligned}
& \text { where } \\
& \begin{aligned}
& \mathcal{L}_{q b}[\rho]=\gamma_{q b}\left[N\left(\omega_{q}\right)+1\right]\left[\sigma_{-} \rho \sigma_{+}-\frac{1}{2} \sigma_{+} \sigma_{-} \rho-\frac{1}{2} \rho \sigma_{+} \sigma_{-}\right] \\
&+\gamma_{q b} N\left(\omega_{q}\right)\left[\sigma_{+} \rho \sigma_{-}-\frac{1}{2} \sigma_{-} \sigma_{+} \rho-\frac{1}{2} \rho \sigma_{-} \sigma_{+}\right],
\end{aligned} \\
& \begin{aligned}
\mathcal{L}_{r} \rho= & \gamma_{r}\left[N\left(\omega_{r}\right)+1\right]\left[a \rho a^{\dagger}-\frac{1}{2} a^{\dagger} a \rho-\frac{1}{2} \rho a^{\dagger} a\right] \\
& +\gamma_{r} N\left(\omega_{r}\right)\left[a^{\dagger} \rho a-\frac{1}{2} a a^{\dagger} \rho-\frac{1}{2} \rho a a^{\dagger}\right],
\end{aligned}
\end{aligned}
$$

where $\gamma_{q b}$ and $\gamma_{r}$ are the decay rates of the qubit and the resonator, respectively. $N(\omega)=1 /[\exp (\beta \hbar \omega)-1]$ is the average occupation number of the bath mode with the frequency $\omega$ at the temperature $T$ with $\beta=1 /\left(k_{B} T\right)$. In the experiment, measurements are taken in a dilution refrigerator at temperature $T \lesssim 20 \mathrm{~m} K$. For $\omega_{q b} / 2 \pi=\omega_{r} / 2 \pi=2.417 \mathrm{GHz}$, the temperature of the bath can taken as 0 , since $N\left(\omega_{q b}\right)$ and $N\left(\omega_{r}\right)$ are less than 0.003 .

In the experiment, the system is initialized in the ground state $|g, 0\rangle$ and a weak, transverse probe field with frequency $\omega$ is applied to detect the spectrum of the coupled system. The detected spectrum intensity is proportional to the integral of the probability $P(t)$ of the qubit being in the excited state over a fixed time interval:

$I \propto \int_{0}^{t} P(t) d t$

where

$P(t)=\frac{1}{2}\left[1+\left\langle\sigma_{z}(t)\right\rangle\right]$

The schematic of the four lowest energy levels of the coupled system is shown Fig. 5a. Due to the coupling between the qubit and the resonator, the lowest two excited states of the system $| \pm\rangle=(|e, 0\rangle \pm|g, 1\rangle) / \sqrt{2}$ are

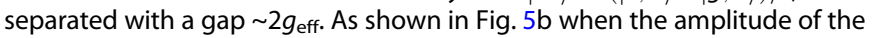
control field $\lambda_{z}=0$, the resonance absorption peaks corresponding to the transitions [denoted by the blue arrows in Fig. 5a] between the ground state $|g, 0\rangle$ and the lowest two excited states $| \pm\rangle$ locate at $\delta=\omega_{a b}-\omega=$ $\pm g$. As the amplitude $\lambda_{z}$ of the control field increases, the gap first decreases and then vanishes at the switch point $\left(\lambda_{z} \approx 1.2 \omega_{z}\right)$, since the coupling between the qubit and the resonator is almost switched off. When $\lambda_{z}>1.2 \omega_{z}$, the coupling is switched on again and the gap increases with $\lambda_{z}$. The coupling between the qubit and the resonator is switched off again when $x=2 \lambda_{z} / \omega_{z}$ reaches the second zero point of the Bessel function $J_{0}(x)$.

When the decay rates of the qubit and the resonator satisfy $\gamma_{q(r)}<g \ll \omega_{q(r)}$, the first two excited states $| \pm\rangle$ form a quasi-invariant subspace. If the system is initially in the state $|e 0\rangle$, a Rabi oscillation induced by the qubit-resonator coupling is observed as shown in Fig. $5 \mathrm{c}$. 

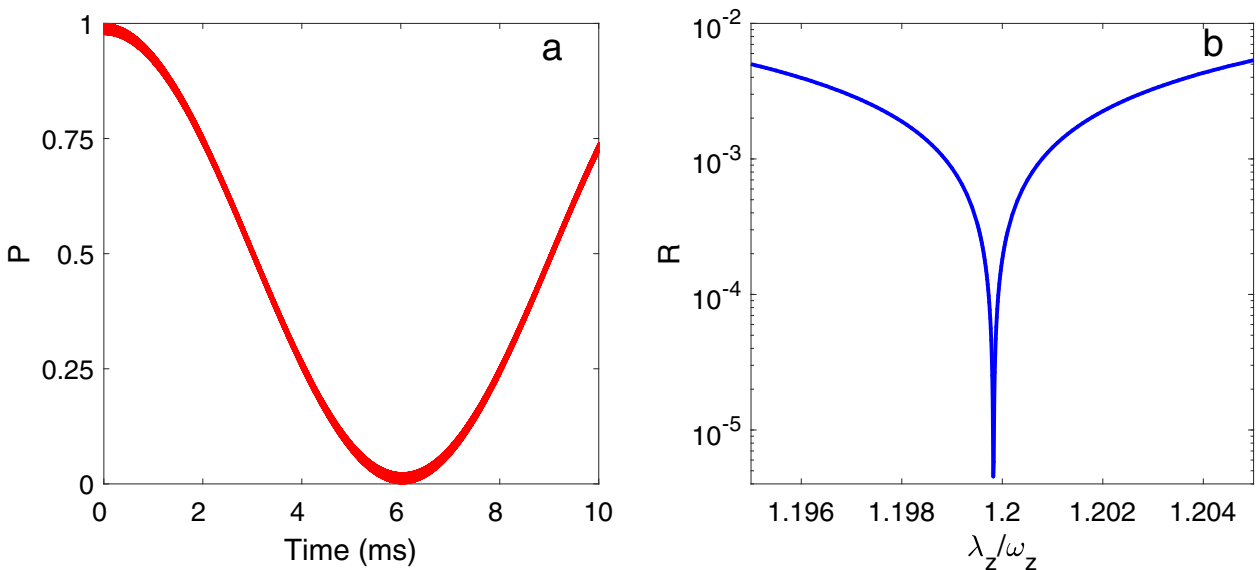

Fig. 6 a The probabilities $P$ of the qubit at the excited state as a function of time. The whole system is initially in the state $|0, e\rangle$ and an effective Rabi oscillation is observed. Here, the coupling strength between the qubit and resonator is $g / 2 \pi=9.14 \mathrm{MHz}$ and the amplitude of the control field is around the switch-off point $\lambda_{z} / \omega_{z} \approx 1.2$. The period of the effective Rabi oscillation is prolonged greatly, i.e., $T_{R, \text { eff }} / T_{R} \approx 4.5 \times$ $10^{-6}$. b The on/off ratio $R$ as a function of $\lambda_{z} / \omega_{z}$ is displayed

When the amplitude of the control field is zero, the period of the Rabi oscillation is $T_{R}=2 \pi / g$. When the strength of the control field increases, the effective coupling strength decreases and the period $T_{R, \text { eff }}$ of the Rabi oscillation becomes longer. At the switch point, the coupling between the qubit and resonator is effectively switched off and the Rabi oscillation disappears.

\section{Mathematical model example}

Assume the system to be initialized in the state $|\psi(0)\rangle=|e\rangle \otimes|0\rangle$ with the qubit in its excited state and the resonator is in the vacuum state.

If no longitudinal control field is applied to the qubit (i.e., $\lambda_{z}=0$ ), the Rabi oscillation between the qubit and the resonator takes place in the single-excitation subspace $\{|e, 0\rangle,|g, 1\rangle\}$. The period of this Rabi oscillation is $T_{R}=\pi / g$. After the control field is applied, the effective coupling between the qubit and the resonator is suppressed and almost eliminated at the switch-off point $\lambda_{z} / \omega_{z} \approx 1.2$. Correspondingly, the period of the effective Rabi oscillation $T_{R, \text { eff }}$ around the switch-off point is prolonged greatly by the control field, as shown in Fig. 6a. Now, we define the on/off ratio as

$R=\frac{T_{R, \text { eff }}}{T_{R}}$.

Numerical simulations show that the period of the effective Rabi oscillation changes drastically near the switch-off point [see Fig. 6a]. The on/off ratio $R$ as a function of the amplitude $\lambda_{z}$ of the control field is displayed in Fig. 6b. We find that $R$ decreases drastically when $\lambda_{z}$ approaches the switch-off point $\lambda_{z} \approx 1.2 \omega_{z}$, which is slightly smaller than the one that matches the zero point of the Bessel function $J_{0}\left(2 \lambda_{z} / \omega_{z}\right)$. So if the amplitude $\lambda_{z}$ of the control field can be precisely manipulated, the coupling between the qubit and the resonator can be effectively switched off by the longitudinal field, i.e., $R<10^{-5}$.

\section{DATA AVAILABILITY}

The datasets generated during and/or analysed during the current study are available from the corresponding author on reasonable request. The datasets are also available in the figshare.com repository, [https://figshare.com/articles/ An_efficient_and_compact_switch_for_quantum_circuits_data/6726965].

\section{ACKNOWLEDGMENTS}

We thank the Laboratory of Microfabrication, Institute of Physics CAS, and National Center for Nanoscience and Technology for the support of the sample fabrication. $X$ B.Z., Y.X.L., and D.N.Z. are supported by the National Basic Research Program (973) of China under Grant no. 2017YFA0304300. Y.X.L., D.N.Z., and C.P.S. are also supported under Grant No. 2014CB921400. X.B.Z. is supported by NSFC under Grant no. 11574380, the Chinese Academy of Science, Alibaba Cloud and Science and Technology Committee of Shanghai Municipality. K.N. acknowledges support from the Japanese MEXT Grant-in-Aid for Scientific Research on Innovative Areas "Science of hybrid quantum systems" (Grant no. 2703). Y.X.L. and D.N.Z. are also supported by NSFC under Grant no. 91321208. We acknowledge Haohua Wang and Kai Xu at Zhejiang University for providing the technical support on the qubit-qubit device and its measurement setup in this experiment.

\section{AUTHOR CONTRIBUTIONS}

Y.X.L. and X.B.Z. conceived the study. X.B.Z. and Y.W. designed the experiment. Y.W., X.B.Z., and Y.R.Z. designed the sample. H.D., X.B.Z., Z.G.Y., and Y.J.Z. fabricated the sample. Y.W., M.G., and Y.R.Z. carried out the measurements and data analysis with $X$. B.Z. and Y.X.L. providing supervision. Y.X.L. developed the theoretical mode. L.P.Y. performed theoretical simulations and wrote the supplementary material under the guide of Y.X.L. and C.P.S. D.N.Z. and L.L. advised on the experiment. W.J.M. and K.N. provided theoretical support. Y.X.L., Y.W., X.B.Z., A.D.C., and W.J.M. wrote the manuscript with contributions from all authors. X.B.Z. and Y.X.L. designed and supervised the project.

\section{ADDITIONAL INFORMATION}

Competing interests: The authors declare no competing interests.

Publisher's note: Springer Nature remains neutral with regard to jurisdictional claims in published maps and institutional affiliations.

\section{REFERENCES}

1. Ladd, T. D. et al. Quantum computers. Nature 464, 45 (2010).

2. Principles and Methods of Quantum Information Technologies. (eds. Yamamoto, Y. \& Semba, K.) (Springer, Berlin, 2016).

3. You, J. Q. \& Nori, F. Superconducting circuits and quantum information. Phys. Today 58, 42 (2005).

4. Devoret, M. H. \& Schoelkopf, R. J. Superconducting circuits for quantum information: an outlook. Science 339, 1169 (2013).

5. Barends, R. et al. Digitized adiabatic quantum computing with a superconducting circuit. Nature 534, 222 (2016).

6. Berkley, A. J. et al. Entangled macroscopic quantum states in two superconducting qubits. Science 300, 1548 (2003).

7. McDermott, R. et al. Simultaneous state measurement of coupled josephson phase qubits. Science 307, 1299 (2005).

8. Blais, A., van den Brink, A. M. \& Zagoskin, A. M. Tunable coupling of superconducting qubits. Phys. Rev. Lett. 90, 127901 (2003).

9. Cleland, A. N. \& Geller, M. R. Superconducting qubit storage and entanglement with nanomechanical resonators. Phys. Rev. Lett. 93, 070501 (2004).

10. Sillanpaa, M. A., Park, J. I. \& Simmonds, R. W. Coherent quantum state storage and transfer between two phase qubits via a resonant cavity. Nature 449, 438 (2007).

11. Majer, J. et al. Coupling superconducting qubits via a cavity bus. Nature 449,443 (2007) 
12. Eichler, C. et al. Observation of entanglement between itinerant microwave photons and a superconducting qubit. Phys. Rev. Lett. 109, 240501 (2012).

13. Beaudoin, F., da Silva, M. P., Dutton, Z. \& Blais, A. First-order sidebands in circuit QED using qubit frequency modulation. Phys. Rev. A 86, 022305 (2012).

14. Strand, J. D. et al. First-order sideband transitions with flux-driven asymmetric transmon qubits. Phys. Rev. B 87, 220505 (2013).

15. Fedorov, A., Steffen, L., Baur, M., da Silva, M. P. \& Wallraff, A. Implementation of a Toffoli gate with superconducting circuits. Nature 481, 170 (2012).

16. Reed, M. D. et al. Realization of three-qubit quantum error correction with superconducting circuits. Nature 482, 382 (2012).

17. Corcoles, A. D. et al. Demonstration of a quantum error detection code using a square lattice of four superconducting qubits. Nat. Commun. 6, 6979 (2015).

18. Neeley, M. et al. Generation of three-qubit entangled states using superconducting phase qubits. Nature 467, 570 (2010).

19. Mariantoni, M. et al. Implementing the quantum von Neumann architecture with superconducting circuits. Science 334, 61 (2011).

20. Dicarlo, L. et al. Preparation and measurement of three-qubit entanglement in a superconducting circuit. Nature 467, 574 (2011).

21. Barends, R. et al. Superconducting quantum circuits at the surface code threshold for fault tolerance. Nature 508, 500 (2014).

22. Kelly, J. et al. State preservation by repetitive error detection in a superconducting quantum circuit. Nature 519, 66 (2015).

23. Risté, D. et al. Detecting bit-flip errors in a logical qubit using stabilizer measurements. Nat. Commun. 6, 6983 (2015).

24. Saira, O. P. et al. Entanglement genesis by ancilla-based parity measurement in 2D circuit QED. Phys. Rev. Lett. 112, 070502 (2014).

25. Zheng, Y. et al. Solving systems of linear equations with a superconducting quantum processor. Phys. Rev. Lett. 118, 210504 (2017).

26. Song, C. et al. 10-Qubit entanglement and parallel logic operations with a superconducting circuit. Phys. Rev. Lett. 119, 180511 (2017).

27. Rigetti, C., Blais, A. \& Devoret, M. Protocol for universal gates in optimally biased superconducting qubits. Phys. Rev. Lett. 94, 240502 (2005).

28. Liu, Y. X., Wei, L. F., Tsai, J. S. \& Nori, F. Controllable coupling between flux qubits. Phys. Rev. Lett. 96, 067003 (2006).

29. Hime, T. et al. Solid-state qubits with current-controlled coupling. Science 314, 1427 (2006)

30. Niskanen, A. O. et al. Quantum coherent tunable coupling of superconducting qubits. Science 316, 723 (2007).

31. van der Ploeg, S. H. W. et al. Controllable coupling of superconducting flux qubits. Phys. Rev. Lett. 98, 057004 (2007).

32. Harris, R. et al. Sign- and magnitude-tunable coupler for superconducting flux qubits. Phys. Rev. Lett. 98, 177001 (2007).

33. Bialczak, R. C. et al. Fast tunable coupler for superconducting qubits. Phys. Rev. Lett. 106, 060501 (2011)

34. Allman, M. S. et al. Tunable resonant and nonresonant interactions between a phase qubit and LC resonator. Phys. Rev. Lett. 112, 123601 (2014).

35. Srinivasan, S. J., Hoffman, A. J., Gambetta, J. M. \& Houck, A. A. Tunable coupling in circuit quantum electrodynamics using a superconducting charge qubit with a Vshaped energy level diagram. Phys. Rev. Lett. 106, 083601 (2011).

36. Baust, A. et al. Tunable and switchable coupling between two superconducting resonators. Phys. Rev. B 91, 014515 (2015).

37. Pinto, R. A., Korotkov, A. N., Geller, M. R., Shumeiko, V. S. \& Martinis, J. M. Analysis of a tunable coupler for superconducting phase qubits. Phys. Rev. B 82, 104522 (2010).

38. Gambetta, J. M., Houck, A. A. \& Blais, A. Superconducting qubit with purcell protection and tunable coupling. Phys. Rev. Lett. 106, 030502 (2011).

39. Hoffman, A. J., Srinivasan, S. J., Gambetta, J. M. \& Houck, A. A. Coherent control of a superconducting qubit with dynamically tunable qubit-cavity coupling. Phys. Rev. B 84, 184515 (2011).

40. Chen, Y. et al. Qubit architecture with high coherence and fast tunable coupling. Phys. Rev. Lett. 113, 220502 (2014).

41. Chow, J. M. et al. Simple all-microwave entangling gate for fixed-frequency superconducting qubits. Phys. Rev. Lett. 107, 080502 (2011).

42. Geller, M. R. et al. Tunable coupler for superconducting Xmon qubits: perturbative nonlinear model. Phys. Rev. A 92, 012320 (2015).
43. Weber, S. J. et al. Coherent coupled qubits for quantum annealing. Phys. Rev. Appl. 8, 014004 (2017).

44. Allman, M. S. et al. rf-SQUID-mediated coherent tunable coupling between a superconducting phase qubit and a lumped-element resonator. Phys. Rev. Lett. 104, 177004 (2010).

45. Bourassa, J. et al. Ultrastrong coupling regime of cavity QED with phase-biased flux qubits. Phys. Rev. A 80, 032109 (2009).

46. Mariantoni, M. et al. Two-resonator circuit quantum electrodynamics: a superconducting quantum switch. Phys. Rev. B 78, 104508 (2008).

47. Reuther, G. M. et al. Two-resonator circuit quantum electrodynamics: dissipative theory. Phys. Rev. B 81, 144510 (2010).

48. Zhou, L., Yang, S., Liu, Y. X., Sun, C. P. \& Nori, F. Quantum Zeno switch for singlephoton coherent transport. Phys. Rev. A 80, 062109 (2009).

49. Liu, Y. X., Yang, C. X., Sun, H. C. \& Wang, X. B. Coexistence of single- and multiphoton processes due to longitudinal couplings between superconducting flux qubits and external fields. New J. Phys. 16, 015031 (2014).

50. Agarwal, G. S. Control of decoherence and relaxation by frequency modulation of a heat bath. Phys. Rev. A 61, 013809 (1999).

51. Agarwal, G. S. \& Harshawardha, W. Realization of trapping in a two-level system with frequency-modulated fields. Phys. Rev. A 50, R4465 (1994).

52. Koch, J. et al. Charge-insensitive qubit design derived from the Cooper pair box. Phys. Rev. A 76, 042319 (2007).

53. Barends, R. et al. Coherent Josephson qubit suitable for scalable quantum integrated circuits. Phys. Rev. Lett. 111, 080502 (2013).

54. van der Wal, C. H. et al. Quantum superposition of macroscopic persistent-current states. Science 290, 773 (2000).

55. Chiorescu, I. et al. Coherent dynamics of a flux qubit coupled to a harmonic oscillator. Nature 431, 159 (2004).

56. Li, J. et al. Motional averaging in a superconducting qubit. Nat. Commun. 4, 1420 (2013).

57. Wilson, C. M. et al. Coherence times of dressed states of a superconducting qubit under extreme driving. Phys. Rev. Lett. 98, 257003 (2007).

58. Wilson, C. M. et al. Dressed relaxation and dephasing in a strongly driven twolevel system. Phys. Rev. B 81, 024520 (2010).

59. Didier, N., Bourassa, J. \& Blais, A. Fast quantum nondemolition readout by parametric modulation of longitudinal qubit-oscillator interaction. Phys. Rev. Lett. 115, 203601 (2015).

60. Yan, Y., Lu, Z., Zheng, H. \& Zhao, Y. Exotic fluorescence spectrum of a superconducting qubit driven simultaneously by longitudinal and transversal fields. Phys. Rev. A 93, 033812 (2016).

61. Silveri, M. P., Tuorila, J. A., Thuneberg, E. V. \& Paraoanu, G. S. Quantum systems under frequency modulation. Rep. Prog. Phys. 80, 056002 (2017).

62. Billangeon, P.-M., Tsai, J. S. \& Nakamura, Y. Scalable architecture for quantum information processing with superconducting flux qubits based on purely longitudinal interactions. Phys. Rev. B 92, 020509 (2015).

63. Richer, S., Maleeva, N., Skacel, S. T., Pop, I. M. \& DiVincenzo, D. Inductively shunted transmon qubit with tunable transverse and longitudinal coupling. Phys. Rev. $B$ 96, 174520 (2017)

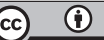

Open Access This article is licensed under a Creative Commons Attribution 4.0 International License, which permits use, sharing, adaptation, distribution and reproduction in any medium or format, as long as you give appropriate credit to the original author(s) and the source, provide a link to the Creative Commons license, and indicate if changes were made. The images or other third party material in this article are included in the article's Creative Commons license, unless indicated otherwise in a credit line to the material. If material is not included in the article's Creative Commons license and your intended use is not permitted by statutory regulation or exceeds the permitted use, you will need to obtain permission directly from the copyright holder. To view a copy of this license, visit http://creativecommons. org/licenses/by/4.0/.

(c) The Author(s) 2018 